\title{
Science vs. technology in radiation therapy from X-rays to ions
}

\author{
Eugene Surdutovich ${ }^{1 *}$ and Andrey V Solov'yov ${ }^{2}$
}

\author{
*Correspondence: \\ surdutov@oakland.edu \\ ${ }^{1}$ Department of Physics, \\ Oakland University, 146 \\ Library Drive, Rochester, MI \\ 48309, USA \\ Full list of author information \\ is available at the end of the \\ article
}

In general, science and technology march in their ways, sometimes starting at different times and developing at different paces. Radiation therapy as an application of ionizing radiation to treat tumors by inactivating cells is a good example of this notion. Brachytherapy, a cancer treatment performed by placing a radioactive source in the direct vicinity of the tumor, was independently suggested by Curie and Bell (Gupta 1995) within 5 years of the discovery of radioactivity by Becquerel in 1896. This happened well before the radioactivity and the propagation of different types of projectiles in tissue were understood. Then, the development of science helped to better select the isotopes and their dosage to improve brachytherapy.

Treatments of tumors with X-rays started at the end of the 1890s, soon after the discovery of X-rays by Roentgen in 1895 . One of the pioneering works on radiation damage was done in 1930s (Timofeeff-Ressovsky et al. 1935), but the first theory of biodamage with X-rays was suggested by Lea (1955) who in 1955 had very little idea about DNA, not even speaking about the types of lesions. Nevertheless, by the end of 1990s, the radiation damage due to exposure to X-rays was understood in detail (Hall and Giaccia 2012). Current improvements and modifications to photon therapy, such as FLASH therapy (Montay-Gruel et al. 2018), arise from biological rather than physical or chemical properties.

Hadron therapy is developing through similar stages. Proton therapy was first suggested in 1946 by Wilson and the first cancer treatment took place in 1954. Therapy was developed in different experimental facilities until the first clinical center was built in 1990 in Loma Linda, CA. The understanding of differences between radiation damage with ions and X-rays from the theoretical point of view (besides the advantages of depth dose curves such as the Bragg peak that has been known much earlier (Ma and Lomax 2012) is present in the paper of Butts and Katz (1967), which became a seminal paper of Katz's theory. In that paper, the idea of targeting of bacteria with $\delta$-electrons was considered and the concept of radial dose (the distribution of dose as a function of distance from ion's path) was emphasized.

However, the treatment planning developed in 1990s is based on microdosimetry (Rossi and Zaider 1996) and on models that were derived from the assessments of radiation damage done with X-rays. For instance, the modified microdosimetric-kinetic model (Kase et al. 2006), which is one of the pillars of contemporary treatment planning, is a modification of the microdosimetric-kinetic model (Hawkins 1996) that was derived 
from previous models obtaining cell survival curves (dependence of probability of cell survival on dose) for "any" value of the linear energy transfer (LET). This model, being mathematically sound, is based on microdosimetry and takes into account a mathematical model for enzymatic repair.

The local effect model (LEM) (Scholz and Kraft 1996) appeared as a hybrid that has a radial dose as one of the components, but the probability of lethal damage is obtained from the X-ray survival curves. This model has undergone several modifications, the LEM IV (Friedrich et al. 2012) being considerably different from the first three (Bueve 2017). In spite of significant empirically established improvements of LEM IV with respect to LEM I, the latter is still being used in clinical treatment planning.

Since 2009, the multiscale approach (MSA) to the physics of ion-beam therapy has been developed (Solov'yov et al. 2009; Surdutovich and Solov'yov 2014; Solov'yov 2017). This approach set the goal of building a comprehensive scenario of radiation damage with ions including all relevant physical, chemical and biological effects. MSA elaborated the targeting aspects of Katz's theory considering the energy deposition by secondary electrons and formation of reactive species. Then, the physical stage of the scenario was reconsidered. First, the effect of low-energy electrons whose action was discovered by Sanche and his group (2005) was taken into account. In addition to the direct damage done by secondary electrons, ion-induced shock waves were predicted. This prediction is based on the idea that the pressure inside a nm-size region around ion paths can develop faster than the energy could be transferred away from that region. The ion-induced shock waves can substantially affect the initial conditions for the chemical stage of the scenario.

This series contains three reviews and one research paper. The main paper that has nearly the same title as the series, "Multiscale modelling for cancer radiotherapies" (Surdutovich and Solov'yov 2019), is a general review of the MSA. The review by de Vera et al. (2019) is devoted to ion-induced shock waves in the context of ionbeam therapy. The review by Baldacchino et al. (2019) is devoted to the chemical stage of the radiation damage scenario. Finally, the research paper by Verkhovtsev et al. (2019) presents a new analysis of survival curves predicted by the MSA for healthy tissue cells, which is a continuation of previous research (Surdutovich and Solov'yov 2014; Verkhovtsev et al. 2016). It also contains the MSA treatment of the relative biological effectiveness including the overkill effect that appears there naturally, without any special modifications.

What is the right path to the future of optimization and planning of ion-beam therapy? Being biased as the authors of the multiscale approach, we believe that the MSA, based on a solid theoretical ground and inclusive with respect to physical, chemical and biological aspects, can be a part of this future. Certainly, the track structure community (Goodhead et al. 1993; Deingfelder 2006; Friedland et al. 2017; Nikjoo et al. 1998, 2006; Liamsuwan and Nikjoo 2013; Frese et al. 2012; Stewart 2015; McNamara et al. 2017) simulating the scenario of radiation damage and going through physical, chemical, and biological stages also aim at constructing a scientific approach to treatment planning. Do their Monte Carlo simulations include "everything" they have to include to cover the relevant science? Time will give the answer to the above question, but now we hope that more people ask what science is included in the current clinical protocols and how to improve them. 


\section{Authors' contributions}

ES and AVS discussed the editorial, ES drafted the manuscript. Both authors read and approved the final manuscript.

\section{Competing interests}

The authors declare that they have no competing interests.

\section{Author details}

${ }^{1}$ Department of Physics, Oakland University, 146 Library Drive, Rochester, MI 48309, USA. ${ }^{2}$ MBN Research Center, Altenhöferallee 3, 60438 Frankfurt am Main, Germany.

Received: 21 October 2019 Accepted: 21 October 2019

Published online: 31 October 2019

\section{References}

Baldacchino G, Brun E, Denden I, Bouhadoun S, Roux R, Khodja H, Sicard-Rocelli C. Importance of radiolytic reactions during high-let irradiation modalities: let effect, role of $\mathrm{O}_{2}$ and radiosensitization by nanoparticles. Cancer Nano. 2019;10:3.

Bueve M. Biophysics modeling to optimize ion beam cancer therapy. In: Solov'yov AV, editor. Nanoscale insights into ionbeam cancer therapy. Cham: Springer; 2017.

Butts JJ, Katz R. Theory of rbe for heavy ion bombardment of dry enzymes and viruses. Radiat Res. 1967;30:855-71.

de Vera P, Surdutovich E, Solov'yov AV. The role of shock waves on the biodamage induced by ion beam radiation. Cancer Nano. 2019:10:5.

Deingfelder M. Track structure: time evolution from physics to chemistry. Radiat Prot Dosim. 2006;122:16-21.

Frese MC, Yu VK, Stewart RD, Carlson DJ. A mechanism-based approach to predict the relative biological effectiveness of protons and carbon ions in radiation therapy. Int J Radiat Oncol. 2012;83:442-50.

Friedland W, Schmitt E, Kundrat P, Dingfelder M, Baiocco G, Barbieri S, Ottolenghi A. Comprehensive track-structure based evaluation of dna damage by light ions from radiotherapy-relevant energies down to stopping. Sci Rep. 2017;7:45161.

Friedrich T, Scholz U, Elsässer T, Durante M, Scholz M. Calculation of the biological effects of ion beams based on the microscopic spatial damage distribution pattern. Int J Radiat Biol. 2012;88:103-7.

Goodhead DT, Thacker J, Cox R. Effects of radiations of different qualities on cells: molecular mechanisms of damage and repair. Int J Radiat Biol. 1993;63:543-56.

Gupta VK. Brachytherapy_ - past, present and future. J Med Phys. 1995;20:31-8.

Hall EJ, Giaccia AJ. Radiobiology for radiologist. Baltimore: Lippincott Williams \& Wilkins; 2012

Hawkins RB. A microdosimetric-kinetic model of cell death from exposure to ionizing radiation of any LET, with experimental and clinical applications. Int J Radiat Biol. 1996;69:739-55.

Kase Y, Kanai T, Matsumoto Y, Furusawa Y, Okamoto H, Asaba T, Sakama M, Shinoda H. Microdosimetric measurements and estimation of human cell survival for heavy-ion beams. Radiat Res. 2006;166:62938.

Lea DE. Actions of radiation on living cells. 2nd ed. New York: Cambridge Univ. Press; 1955.

Liamsuwan T, Nikjoo H. A monte carlo track structure simulation code for the full-slowing-down carbon projectiles of energies 1 kev/u-10 mev/u in water. Phys Med Biol. 2013;58:673.

Ma C-MC, Lomax T. Proton and carbon ion therapy. Boca Raton: CRC Press; 2012.

McNamara A, Geng C, Turner R, Mendez JR, Perl J, Held K, Faddegon B, Paganetti H, Schuemann J. Validation of the radiobiology toolkit topas-nbio in simple dna geometries. Phys Med. 2017;33:207-15.

Montay-Gruel P, Bouchet A, Jaccard M, Patin D, Serduc R, Aim W, Petersson K, Petit B, Bailat C, Bourhis J, Bräuer-Krisch E, Vozenin M-C. X-rays can trigger the flash effect: Ultra-high dose-rate synchrotron light source prevents normal brain injury after whole brain irradiation in mice. Radiot Oncol. 2018;129:582-8.

Nikjoo H, Uehara S, Wilson WE, Hoshi M, Goodhead DT. Track structure in radiation biology: theory and applications. Int J Radiat Biol. 1998;73:355-64.

Nikjoo H, Uehara S, Emfietzoglou D, Cucinotta FA. Track-structure codes in radiation research. Radiat Meas. 2006;41:1052.

Rossi HH, Zaider M. Microdosimetry and its applications. Berlin: Springer; 1996.

Sanche L. Low energy electron-driven damage in biomolecules. Eur Phys J D. 2005:35:367-90.

Scholz M, Kraft G. Track structure and the calculation of biological effects of heavy charged particles. Adv Space Res. 1996:18:5-14.

Solov'yov AV. Nanoscale insights into ion-beam cancer therapy. Cham: Springer; 2017.

Solov'yov AV, Surdutovich E, Scifoni E, Mishustin I, Greiner W. Physics of ion beam cancer therapy: a multiscale approach. Phys Rev E. 2009;79:011909.

Stewart RD, et al. Simulation of DNA Double Strand Break (DSB) relative biological effectiveness (RBE) for photons, neutrons, and light ions. Phys Med Biol. 2015;60:8249-74.

Surdutovich E, Solov'yov AV. Multiscale approach to the physics of radiation damage with ions. Eur Phys J D. 2014;68:353.

Surdutovich E, Solov'yov AV. Multiscale modelling for cancer radiotherapies. Cancer Nano. 2019;10:6.

Timofeeff-Ressovsky NW, Zimmer KG, Delbrück M. Über die natur der genmutation und der genstruktur. Nachr Ges Wiss. Göttingen. 1935;1:189-245.

Verkhovtsev A, Surdutovich E, Solov'yov AV. Multiscale approach predictions for biological outcomes in ion-beam cancer therapy. Sci Rep. 2016;6:27654.

Verkhovtsev A, Surdutovich E, Solov'yov AV. Phenomenon-based evaluation of relative biological effectiveness of ion beams by means of the multiscale approach. Cancer Nano. 2019;10:4

\section{Publisher's Note}

Springer Nature remains neutral with regard to jurisdictional claims in published maps and institutional affiliations. 